\title{
AVALIAÇÃO DO ESTADO NUTRICIONAL E DO DESENVOLVIMENTO DA BANANEIRA- PRATA-ANÃ (Musa spp.) EM FUNÇÃO DA ADUBAÇÃO NITROGENADA ${ }^{1}$
}

\author{
PATRÍCIA SOARES FURNO FONTES², ALMY JUNIOR CORDEIRO DE CARVALHO ${ }^{3}$, BRUNO SALES CEREJA4, \\ CLÁUDIA SALES MARINHO ${ }^{3}$, PEDRO HENRIQUE MONNERAT ${ }^{3}$
}

\begin{abstract}
RESUMO - O experimento foi instalado num Argissolo Amarelo, no município de Itaocara-RJ (Latitude de $21^{\circ} 39^{\prime} 12^{\prime}$ 'Sul e Longitude de 42 04 ' $36^{\prime \prime}$ Oeste, com clima conforme Köppen, classificado como Awi, temperatura média anual em torno de $22,5^{\circ} \mathrm{C}$ e precipitação anual de $1041 \mathrm{~mm}$ ), com o objetivo de avaliar o estado nutricional e o desenvolvimento da bananeira cultivar Prata-Anã em função da aplicação de cinco doses de nitrogênio (0; $150 ; 300 ; 450$ e $600 \mathrm{~kg} \mathrm{ha}^{-1} \mathrm{ano}^{-1} \mathrm{de} \mathrm{N}$ ). Os teores dos nutrientes na matéria seca foliar não foram alterados pelas doses de N, exceções feitas ao Mn e ao $\mathrm{Cl}$. Os teores foliares dos nutrientes variaram entre as épocas analisadas, com exceção do Ca. Não houve diferença nos teores de $\mathrm{N}$ na folha analisada, em função do incremento da adubação nitrogenada. Foram encontrados menores teores de K na matéria seca foliar, na época da inflorescência e formação do cacho. A adubação nitrogenada não afetou a época de florescimento e a altura do cacho. Observou-se diminuição na emissão de filhotes com o incremento das doses de $\mathrm{N}$.

Termos para indexação: banana, Musa, nitrogênio, nutrientes foliares, uréia.

\section{EVALUATION OF THE NUTRICIONAL STATUS AND DEVELOPMENT OF BANANA CULTIVAR "PRATA ANÃ" (Musa spp.) IN RELATION TO NITROGEN FERTILIZATION}

\begin{abstract}
The experiment was carried in Yellow Podzolic at Itaocara, Rio de Janeiro State, Brazil, (Latitude 21 ${ }^{\circ} 39^{\prime} 12^{\prime \prime}$ South and Longitude 42 04' 36" West, with climate, conform Köppen, classified as Awi,), to evaluate the nutritional status and the development of the banana cultivar "Prata Anã" in relation to five nitrogen fertilization levels $\left(0,150,300,450\right.$ and $600 \mathrm{~kg} \mathrm{ha}^{-1}$ year ${ }^{-1}$ of $\left.\mathrm{N}\right)$. In general, the analyzed nutrients contents in the leaf dry matter were not modified by the increase of $\mathrm{N}$ dosage, being exception $\mathrm{Mn}$ and $\mathrm{Cl}$. The levels of leaf nutrients differed between sampling seasons, with the exception of $\mathrm{Ca}$. There was no variation in the levels of $\mathrm{N}$ in the leaf dry matter, in relation to nitrogen fertilization. Lower levels of $\mathrm{K}$ were observed in the leaf dry matter during the blooming and bunch development. In the first growing cycle, the nitrogen fertilization had no effect either on blooming time or in the bunch height. There was a decrease in the number of offshoots with the increasing of $\mathrm{N}$ levels.
\end{abstract}

Index terms: banana, Musa, fertilization, nitrogen, leaf nutrient status, urea

\section{INTRODUÇÃO}

A bananeira é uma planta exigente em nutrientes, não só por produzir grande massa vegetativa, mas também por apresentar elevadas quantidades de elementos absorvidos pela planta e exportados pelos frutos (Silva et al., 1999).

Na diagnose foliar de bananeiras, devem ser levados em consideração os diversos fatores que interferem nos teores dos nutrientes nas folhas, num dado momento, e, segundo Martin-Prével (1977), são fatores de origem interna, tais como: cultivar, estádio fenológico das plantas, posição e porção das folhas, além de fatores externos, como clima, solo, parasitismos e tratos culturais.

Os níveis adequados de nutrientes encontrados pela análise foliar ainda não foram estabelecidos para a bananeira. Porém, alguns autores admitem faixas adequadas para macros e micronutrientes, nas condições em que foram determinadas, observando-se grande variação nas informações apresentadas (Tabela 1).

O N tem grande importância do início do desenvolvimento até a emissão da inflorescência, havendo uma redução da sua absorção até a colheita (Martin-Prével, 1977). Segundo Lahav e Turner (1983), em ordem decrescente, a bananeira absorve os seguintes nutrientes: $\mathrm{K}>\mathrm{N}>\mathrm{Ca}>\mathrm{Mg}>\mathrm{S}>\mathrm{P}>\mathrm{Cl}>\mathrm{Mn}>\mathrm{Fe}>\mathrm{Zn}>\mathrm{B}>\mathrm{Cu}$.

O nitrogênio é muito importante para a bananicultura, atuando sobre o crescimento vegetativo, emissão dos rebentos, além de aumentar a quantidade de matéria seca (Borges et al., 1999). Brasil et al. (2000), estudando adubação nitrogenada e potássica em bananeira, observaram que até os 240 dias de plantio, apenas o $\mathrm{N}$ influenciou a circunferência do pseudocaule e altura da planta. Silva et al. (2000), trabalhando com adubação nitrogenada na cultivar Terra, observaram que, no primeiro ciclo da cultura, a adubação nitrogenada influenciou a altura da planta, o número de frutos por cacho e diâmetro médio dos frutos, sendo que a maior produtividade foi obtida com $231 \mathrm{~kg} \mathrm{ha}^{-1} \mathrm{ano}^{-1}$.

Os objetivos deste trabalho foram avaliar o estado nutricional e o efeito da aplicação de N no desenvolvimento da bananeira PrataAnã, na região Noroeste do Estado do Rio de Janeiro.

TABELA 1-Faixas de concentrações de nutrientes observadas em folhas de bananeira em diferentes estudos

\begin{tabular}{lcccccc}
\hline & Nutriente & 1 & 2 & 3 & 4 & 5 \\
\hline $\mathrm{N}$ & $\left(\mathrm{g} \mathrm{kg}^{-1}\right)$ & $35-45$ & $28-40$ & 26 & $27-36$ & $27-36$ \\
$\mathrm{P}$ & $\left(\mathrm{g} \mathrm{kg}^{-1}\right)$ & $2-4$ & $2-2,5$ & 2,2 & $1,8-2,7$ & $1,6-2,7$ \\
$\mathrm{~K}$ & $\left(\mathrm{~g} \mathrm{~kg}^{-1}\right)$ & $38-50$ & $31-40$ & 28 & $30-54$ & $32-54$ \\
$\mathrm{Ca}$ & $\left(\mathrm{g} \mathrm{kg}^{-1}\right)$ & $8-15$ & $8-12$ & 6 & $2,5-12$ & $6,6-12$ \\
$\mathrm{Mg}$ & $\left(\mathrm{g} \mathrm{kg}^{-1}\right)$ & $2,5-8$ & $3-4,6$ & 3 & $3-6$ & $2,7-6$ \\
$\mathrm{~S}$ & $\left(\mathrm{~g} \mathrm{~kg}^{-1}\right)$ & $2,5-8$ & $2,3-2,7$ & 2 & $2-3$ & $1,6-3$ \\
$\mathrm{Cl}$ & $\left(\mathrm{g} \mathrm{kg}^{-1}\right)$ & - & $8-9$ & - & - & $9-18$ \\
$\mathrm{Fe}$ & $\left(\mathrm{mg} \mathrm{kg}^{-1}\right)$ & $76-300$ & $70-200$ & 100 & $80-360$ & $80-360$ \\
$\mathrm{Mn}$ & $\left(\mathrm{mg} \mathrm{kg}^{-1}\right)$ & $100-1000$ & $1000-2200$ & 88 & $20-200$ & $200-1800$ \\
$\mathrm{Zn}$ & $\left(\mathrm{mg} \mathrm{kg}^{-1}\right)$ & $20-200$ & $21-35$ & 20 & $20-50$ & $20-50$ \\
$\mathrm{Cu}$ & $\left(\mathrm{mg} \mathrm{kg}^{-1}\right)$ & $6-25$ & $7-20$ & 8 & $6-30$ & $6-30$ \\
$\mathrm{~B}$ & $\left(\mathrm{mg} \mathrm{kg}^{-1}\right)$ & $10-50$ & $20-80$ & 15 & $10-25$ & $10-25$ \\
\hline
\end{tabular}

1) Jones Jr. et al., 1991; 2) Robinson, 1986; 3) Ribeiro et al., 1999; 4) Prezotti, 1992; 5) Teixeira et al., 1997.

\section{MATERIALEMÉTODOS}

O experimento foi instalado em fevereiro de 2000, num Argissolo Amarelo em Itaocara-RJ, Latitude de 21039'12" Sul e Longitude de 4204'36" Oeste, com clima, classificado por Köppen como Awi, temperatura média anual próxima a $22,5^{\circ} \mathrm{C}$ e precipitação anual de $1041 \mathrm{~mm}$ ). O solo apresentava, na camada de 0 a $20 \mathrm{~cm}$, as seguintes características: $\mathrm{pH}$ em $\mathrm{H}_{2} \mathrm{O}(1: 2,5)$

\footnotetext{
'(Trabalho 062/2002). Recebido: 18/04/2002; Aceito para publicação:20/03/2003 - Apoio financeiro: FAPERJ 'Extraído da Tese de Mestrado do primeiro autor apresentada à Universidade Estadual do Norte Fluminense ${ }^{2}$ Eng. Agr., M.Sc., Doutoranda em Produção Vegetal, UENF/CCTA/LFIT, Campos-RJ, CEP 28013-600 ${ }^{3}$ Eng. Agr., D.Sc., Professores, UENF/CCTA/LFIT, Campos-RJ, CEP 28013-600 E-mail: almy@uenf.br ${ }^{4}$ Eng. Agr., Mestrando em Produção Vegetal, UENF/CCTA/LFIT, Campos-RJ, CEP 28013-600
} 
$=5,8 ; \mathrm{P}=8 \mathrm{mg} \mathrm{kg}^{-1} ; \mathrm{Ca}=2,9 \mathrm{cmol} \mathrm{kg}^{-1} ; \mathrm{Mg}=1 \mathrm{cmol} \mathrm{kg}^{-1} ; \mathrm{K}=104 \mathrm{mg} \mathrm{kg}^{-1}$; $\mathrm{Al}=0 ; \mathrm{H}+\mathrm{Al}=2,7 \mathrm{cmol}_{\mathrm{c}} \mathrm{kg}^{-1} ; \mathrm{Fe}=180 \mathrm{mg} \mathrm{kg}^{-1} ; \mathrm{Cu}=1,6 \mathrm{mg} \mathrm{kg}^{-1} ; \mathrm{Zn}=9,4 \mathrm{mg}$ $\mathrm{kg}^{-1} ; \mathrm{Mn}=28,8 \mathrm{mg} \mathrm{kg}^{-1} ; \mathrm{S}=10,3 \mathrm{mg} \mathrm{kg}^{-1} ; \mathrm{B}=0,20 \mathrm{mg} \mathrm{kg}^{-1} ;$ Carbono $=9,1 \mathrm{~g}$ $\mathrm{kg}^{-1}$; Areia fina $=590 \mathrm{~g} \mathrm{~kg}^{-1}$; Areia média $=50 \mathrm{~g} \mathrm{~kg}^{-1}$; Areia grossa $=10 \mathrm{~g} \mathrm{~kg}$ '; Silte $=160 \mathrm{~g} \mathrm{~kg}^{-1}$; Argila $=190 \mathrm{~g} \mathrm{~kg}^{-1}$. As análises do solo seguiram metodologias da Embrapa (1999). Para P, K, Zn, Cu, Fe e Mn utilizou-se o extrator Mehlich-1; para Ca, $\mathrm{Mg}$ e Al utilizou-se o extrator $\mathrm{KCl} 1 \mathrm{M}$. O B foi extraído com água quente e o $\mathrm{S}$ extraído por íons fosfato $\left(500 \mathrm{mg}\right.$ de $\left.\mathrm{P} \mathrm{L}^{-1}\right)$.

O delineamento experimental foi em blocos casualizados $(5 \times 3)$, os tratamentos foram constituídos de cinco doses de nitrogênio $(0 ; 150 ; 300$; 450 e $600 \mathrm{~kg} \mathrm{ha}^{-1}$ ano $^{-1}$ de N) e 3 repetições. Cada parcela era constituída por 24 plantas (famílias), sendo as quatro centrais consideradas úteis. $\mathrm{O}$ adubo nitrogenado utilizado foi a uréia, aplicado bimestralmente em cobertura. Todas as plantas receberam, em cobertura, doses de $45 \mathrm{~g}$ de $\mathrm{K}_{2} \mathrm{O}$ (Cloreto de potássio) a cada dois meses, $50 \mathrm{~g} \mathrm{de} \mathrm{P}_{2} \mathrm{O}_{5}$ (superfosfato simples) a cada seis meses e $50 \mathrm{~g}$ do formulado com micronutriente FTE-BR12, cuja composição, por kg, tinha $18 \mathrm{~g}$ de $\mathrm{B}$; 8, $0 \mathrm{~g}$ de $\mathrm{Cu}$; $30 \mathrm{~g}$ de Fe; $30 \mathrm{~g}$ de $\mathrm{Mn}$; $1,0 \mathrm{~g}$ de Mo; $\mathrm{e}$ $90 \mathrm{~g}$ de Zn.

O plantio foi realizado em fevereiro de 2000 , em espaçamento $3 \times 3$ m e irrigado por microaspersão. Foram feitos os tratos culturais recomendados para a cultura, tais como: desbaste de filhotes a cada mês, deixando três plantas - mãe, filha e neta, eliminação das folhas velhas, manejo de plantas daninhas e controle de pragas e doenças.

Coletou-se a terceira folha a contar do ápice, utilizando-se de 10 cm da parte interna mediana do limbo foliar, eliminando-se a nervura central (Teixeira et al., 1997). As folhas foram secas em estufa com circulação forçada de ar à temperatura de $70^{\circ} \mathrm{C}$, durante 48 horas. Após a secagem, o material foi triturado em moinho (tipo Wiley) com peneira de 20 mesh e armazenado em frascos hermeticamente fechados (Malavolta et al., 1989).


Foram analisados os seguintes elementos: nitrogênio orgânico (Norg); nitrogênio nítrico $\left(\mathrm{NO}_{3}^{-}\right)$; fósforo $(\mathrm{P})$; potássio $(\mathrm{K})$; cálcio $(\mathrm{Ca})$; magnésio $(\mathrm{Mg})$; enxofre $(\mathrm{S})$; cloro $(\mathrm{Cl})$; ferro $(\mathrm{Fe})$; zinco $(\mathrm{Zn})$; cobre $(\mathrm{Cu})$; manganês (Mn), e boro (B). O Norg, o P e o K foram determinados pelos métodos de Nessler, do azul de molibdato e por espectrofotometria de emissão de chama, respectivamente, após submeter o material vegetal à oxidação pela digestão sulfúrica $\left(\mathrm{H}_{2} \mathrm{SO}_{4} \mathrm{eH}_{2} \mathrm{O}_{2}\right)$. Os elementos $\mathrm{Ca}, \mathrm{Mg}, \mathrm{Fe}, \mathrm{Zn}, \mathrm{Cu}$ e Mn foram determinados por espectrofotometria de absorção atômica, a partir de extratos obtidos após oxidação do material vegetal pela digestão nitroperclórica $\left(\mathrm{HNO}_{3}\right.$ e $\left.\mathrm{HCLO}_{4}\right)$. O B foi determinado colorimetricamente pela azometina- $\mathrm{H}$, após incineração em mufla. $\mathrm{O} \mathrm{NO}_{3}^{-}$e o Cl foram determinados pelo método colorimétrico do ácido salicílico e por titulação com $\mathrm{AgNO}_{3}$, respectivamente, após submeter o tecido vegetal a banho-maria por 1 hora.

Avaliou-se a altura da roseta, tomando-se a distância do nível do solo até $\mathrm{o}$ ângulo formado pelas duas folhas mais velhas; circunferência do pseudocaule a $0,30 \mathrm{~m}$ do solo; número de folhas totais; número de folhas emitidas e número de folhas funcionais, com mais de $50 \%$ de sua superfície verde; número de dias entre o plantio e a emissão da inflorescência ( $1^{\mathrm{a}}$ geração) da planta-mãe; número de filhotes emitidos.

\section{RESULTADOS E DISCUSSÃO}

De modo geral, as médias dos valores observados para os nutrientes estão dentro das faixas encontradas por alguns autores (Tabela 1). Destaca-se apenas que os teores observados para $\mathrm{Zn} \mathrm{e} \mathrm{Cl}$, que foram respectivamente $15,7 \mathrm{mg} \mathrm{kg}^{-1} \mathrm{e} 6,8 \mathrm{~g} \mathrm{~kg}^{-1}$, na planta-mãe (Figura 1) e 14,3 $\mathrm{mg} \mathrm{kg}^{-1}$ e $5,3 \mathrm{~g} \mathrm{~kg}^{-1}$, na planta-filha (Figura 2), ficaram com as médias abaixo das faixas apresentadas por alguns autores (Tabela 1).


out/00 nov/00 dez/00 jan/01 fev/01 Época de amostragem



FIGURA 1- Variação nos teores dos nutrientes na matéria seca foliar (MS) em plantas da $1^{a}$ geração (Planta-mãe) da cultivar de banana-Prata-Anã em função da época de amostragem. Comparação entre épocas feita pelo teste de Tukey, a 5\%. 



FIGURA 2- Variação nos teores dos nutrientes na matéria seca foliar (MS) em plantas da $2^{a}$ geração (Planta-filha) da cultivar de banana-Prata-Anã, em função da época de amostragem. Comparação entre épocas feita pelo teste de Tukey, a 5\%.

Não houve diferenças significativas nos teores de Norg e nitrato na matéria seca das folhas amostradas, em nenhuma época, em função do incremento na dose do adubo nitrogenado. Isto pode sugerir perdas por lixiviação e/ou volatilização do N, ou devido ao comprimento do sistema radicular, que pode atingir em torno de $10 \mathrm{~m} \mathrm{e}$, assim, a dose de $\mathrm{N}$ aplicada num tratamento poderia ter sido absorvida por outro tratamento, igualando os resultados. É possível, ainda, que a folha coletada para amostragem não tenha apresentado variação deste nutriente.

Os teores de $\mathrm{N}$ orgânico variaram entre as diferentes épocas analisadas, de 25,8 a 29,8 $\mathrm{g} \mathrm{kg}^{-1}$ na planta-mãe (Figura 1C), e 29,2 $\mathrm{g} \mathrm{kg}^{-1} \mathrm{a}$ $31,6 \mathrm{~g} \mathrm{~kg}^{-1}$ na planta-filha (Figura 2C). Para o nitrato, houve uma variação de $0,44 \mathrm{~g} \mathrm{~kg}^{-1}$ a $0,66 \mathrm{~g} \mathrm{~kg}^{-1}$ na planta-mãe (Figura 1C), e de $0,43 \mathrm{~g} \mathrm{~kg}^{-}$ ${ }^{1}$ a $0,64 \mathrm{~g} \mathrm{~kg}^{-1}$ (Figura 2C) na planta-filha.

$\mathrm{O}$ incremento da adubação nitrogenada não influenciou os teores dos nutrientes analisados, exceções feitas para o Mn, que aumentou nas plantas da $1^{\text {a }}$ e $2^{\text {a }}$ gerações (Figura 3 ) e $\mathrm{Cl}$, que diminuiu nas plantas da $1^{\text {a }}$ geração $\left(\mathrm{Y}=7,4-0,0021 \mathrm{~N} \mathrm{R}^{2}=0,87^{* *}\right)$.

Observou-se um aumento no teor de Mn nas plantas da $1^{\mathrm{a}}$ e $2^{\mathrm{a}}$ gerações de $173 \%$ e $89 \%$, respectivamente, na matéria seca foliar da bananeira, quando se comparou o teor obtido entre a dose mínima e máxima de N (Figura 3). Este aumento pode ser resultado da maior solubilidade do $\mathrm{Mn}$ em função da possível redução do $\mathrm{pH}$ do solo, provocado pelo incremento das doses de N. Verificaram-se, também, aumentos nas concentrações foliares de $\mathrm{Mn}$ nas plantas da $1^{\mathrm{a}}$ geração em relação às épocas amostradas (Figura 1D).

Quanto ao K, verificou-se que, na época da inflorescência e formação do cacho nas plantas da $1^{\text {a }}$ geração (Janeiro e Fevereiro), se encontraram menores teores de $\mathrm{K}$ na matéria seca foliar (Figura 1A). Isto foi ocasionado, provavelmente, pela translocação deste nutriente das folhas para o cacho, que, neste momento, era o dreno mais forte da planta. Segundo Lahav e Turner (1983), o K é o nutriente mais encontrado nos frutos da bananeira. Com isto, sua exigência é maior na época de formação do cacho.

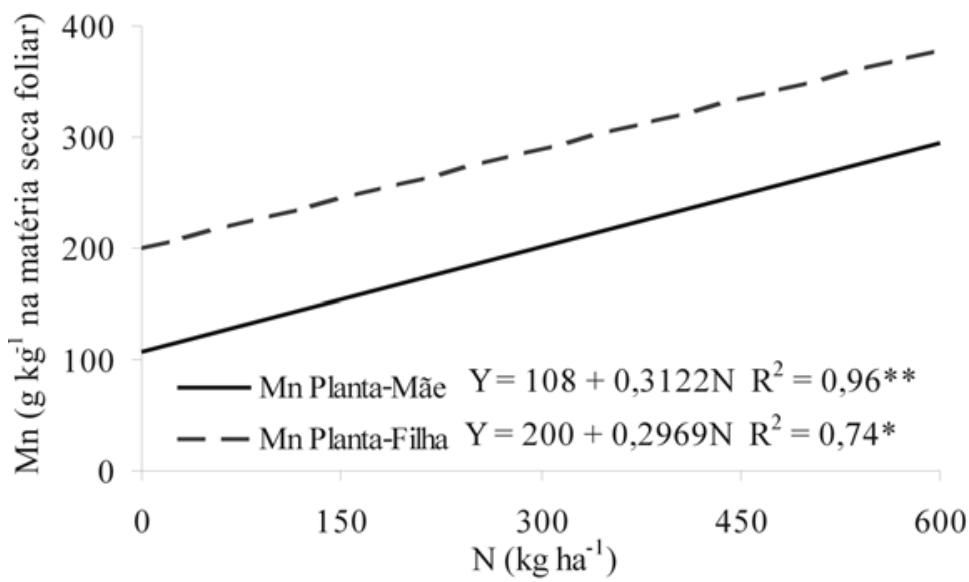

FIGURA 3- Teores de $\mathrm{Mn}\left(\mathrm{mg} \mathrm{kg}^{-1}\right)$ na matéria seca foliar, em duas gerações de cultivo (planta-mãe e planta-filha) da bananeiraPrata-Anã, em função das doses de N.

Os teores dos nutrientes na matéria seca foliar, na bananeiraPrata-Anã, seguiram a seguinte ordem de concentração: (1 $1^{\text {a }}$ geração) $\mathrm{K}>\mathrm{N}>\mathrm{Cl}>\mathrm{Ca}>\mathrm{Mg}>\mathrm{S}>\mathrm{P}>\mathrm{Mn}>\mathrm{Fe}>\mathrm{B}>\mathrm{Zn}>\mathrm{Cu}$; (2 ${ }^{\text {a }}$ geração) $\mathrm{K}>\mathrm{N}>\mathrm{Cl}>\mathrm{Ca}>\mathrm{Mg}>\mathrm{P}>\mathrm{S}>\mathrm{Mn}>\mathrm{Fe}>\mathrm{B}>\mathrm{Zn}>\mathrm{Cu}$ (Figuras 1 e 2).

Os teores dos nutrientes analisados foram influenciados pela época de amostragem, com exceção do Ca na planta-mãe, e Ca e Mg na 
filha, que não apresentaram diferença significativa entre as épocas ( $\mathrm{Fi}-$ gura $1 \mathrm{~A}$ e $2 \mathrm{~A}$ ).

A adubação nitrogenada não afetou o número de dias do plantio ao florescimento e, também, a altura da roseta foliar da $1^{\text {a }}$ geração ou ciclo de cultivo da bananeira-Prata-Anã. Estas duas características apresentaram, respectivamente, médias de 349 dias e $249 \mathrm{~cm}$.

Observaram-se comportamentos semelhantes para plantas da $1^{\mathrm{a} e} 2^{\mathrm{a}}$ gerações, em relação às médias da circunferência do pseudocaule a $0,30 \mathrm{~m}$ do solo, em função das doses de $\mathrm{N}$, tendo sido encontrados pseudocaules de maior circunferência com a dose de $244 \mathrm{~kg} \mathrm{ha}^{-1} \mathrm{ano}^{-1}$ $\operatorname{de~} \mathrm{N}\left(\mathrm{Y}=64,6+0,0536 \mathrm{~N}-0,00011 \mathrm{~N}^{2} \mathrm{R}^{2}=0,85^{* *}\right)$. Comparando-se plantas da $1^{\mathrm{a}}$ e $2^{\mathrm{a}}$ gerações, verificou-se a maior relação altura/circunferência do pseudocaule nas plantas da $2^{\mathrm{a}}$ geração, evidenciando um provável "estiolamento" nestas plantas em relação às da $1^{a}$ geração (Quadro 1).

No primeiro e segundo ciclos, o comportamento foi semelhante para o número de folhas totais (Quadro 1), sendo esta característica influenciada pelo N. Encontrou-se maior $n^{\circ}$ de folhas totais com a dose de $251 \mathrm{~kg} \mathrm{ha}^{-1}$ ano $^{-1} \mathrm{de} \mathrm{N}$.

Observou-se maior número de folhas funcionais nas plantas da $1^{\text {a }}$ geração, possivelmente devido à retirada de folhas pendentes ou doentes que ocorreram em maior quantidade nas plantas da $2^{a}$ geração (Quadro 1); observou-se um efeito quadrático em relação ao incremento de $\mathrm{N}$, com um maior número de folhas funcionais na dose de $255 \mathrm{~kg}$ ha ${ }^{1}$ ano $^{-1}$ para as plantas da $1^{\text {a }}$ e $2^{\text {a }}$ gerações.

Observou-se maior $\mathrm{n}^{\mathrm{o}}$ de folhas emitidas nas plantas da $2^{\mathrm{a}}$ geração (Quadro 1), provavelmente, devido à maior influência do $\mathrm{N}$ na planta-filha, que as recebeu desde o início de seu surgimento, o que não ocorreu com a planta-mãe. Porém, não houve diferença para o $\mathrm{N}^{o} \mathrm{de}$ folhas totais entre as plantas da $1^{\mathrm{a}}$ e $2^{\mathrm{a}}$ gerações, possivelmente, por a planta-mãe ter apresentado maior manutenção das folhas fotossinteticamente ativas por período de tempo maior, ocasionando, assim, maior número de folhas funcionais (Quadro 1).

Observou-se redução na emissão de filhotes da bananeira-Prata-Anã com o aumento na dose de $\mathrm{N}\left(\mathrm{Y}=6,4-0,0024 \mathrm{~N} \mathrm{R}^{2}=0,89^{*}\right)$.

\section{CONCLUSÕES}

1) Os nutrientes analisados não foram influenciados pelo incremento das doses de $\mathrm{N}$, exceções feitas ao $\mathrm{Mn}$, que aumentou, e ao $\mathrm{Cl}$, que reduziu.

2) Houve diferença nos teores foliares dos nutrientes entre as épocas analisadas, exceção feita ao Ca.

3) Os teores dos nutrientes na matéria seca foliar da bananaPrata-Anã, obedeceram à seguinte ordem de concentração: $\mathrm{K}>\mathrm{N}>\mathrm{Cl}>\mathrm{Ca}>\mathrm{Mg}>\mathrm{S}>\mathrm{P}>\mathrm{Mn}>\mathrm{Fe}>\mathrm{B}>\mathrm{Zn}>\mathrm{Cu}$.

4) A adubação nitrogenada não provocou variação no teor de $\mathrm{N}$ na folha analisada.

5) No primeiro ciclo de cultivo, a adubação nitrogenada não afetou a altura da roseta foliar e o intervalo entre o plantio e o florescimento.

6) Houve diminuição na emissão de filhotes com o incremento das doses de $\mathrm{N}$

\section{REFERÊNCIASBIBLIOGRÁFICAS}

BORGES, A.L.; OLIVEIRA, A.M.G.; SOUZA, L.S da. Solos, nutrição e adubação da bananeira. In: ALVES, E.J., Cultura da banana: aspectos técnicos, socioeconômicos e agroindustriais. Cruz das Almas: Embrapa-CNPMF, 1999. p.35-46.

BRASIL. E.C.; OEIRAS,A.H.L.; MENEZES,A.J.E.A. de; VELOSO, C.A.C. Desenvolvimento e produção de frutos de bananeira em resposta à adubação nitrogenada e potássica. Pesquisa Agropecuária Brasileira. v.35, n.12, p.2407-2414, 2000.

EMBRAPA. Manual de análises químicas de solos, plantas e fertilizantes. Brasília: Embrapa Informática Agropecuária, 1999.370p.

JONES JR.; J.B.; WOLF, B.; MILLS, H.A. Plant Analysis Handbook: a practical sampling, preparation, analysis, and interpretation guide. Athens (USA): Micro-Macro Publishing, 1991. 213p.

LAHAV, E.; TURNER, D. Banana nutricion. Bern: Switzerland Potash Institute, 1983.62p. (IPI-Bulletin).

MALAVOLTA, E.; VITTI, G.C.; OLIVEIRA, S.A. de. Avaliação do estado nutricional das plantas: princípios e aplicações. Piracicaba: POTAFOS, 1989. 201p.

MARTIN-PRÉVEL, P. Echantillonnange du bananier por l'analyse foliare; cons'sequences des differences de techiniques. Fruits, Paris, v.32, n.3, p.151-166, 1977.

PREZOTTI, L.C. Recomendação de calagem e adubação para o Estado do Espírito Santo: $3^{\text {a }}$ aproximação. Vitória: EMCAPA, 1992. 73p. (Circular Técnica, 12)

RIBEIRO, A.C.; GUIMARÃES, P.T.G.; ALVAREZ V., V.H. Recomendações para o uso de corretivos e fertilizantes em Minas Gerais: $5^{\text {a }}$ aproximação. Viçosa-MG: CFSEMG, 1999.359p.

ROBINSON, J.B. Fruits, Vines e Nuts. In: REUTER, D.J.; ROBINSON, J.B. (ed). Plant Analysis: an interpretation manual. Melbourne: Inkata Press, 1986. p.120-147.

SILVA, S. de O.; ALVES, E.J.; SHEPHERD, K.; DANTAS, J.L.L. Cultivares. In: ALVES, E.J. A cultura da banana: aspectos técnicos, socioeconômicos e agroindustriais. 2.ed., Brasília: EMBRAPA-SPI, 1999. p.85-106.

SILVA, T.O.; BORGES, A.L.; CALDAS, R.C.; ALMEIDA, I.E. de. Adubação nitrogenada para a bananeira 'Terra'. In: CONGRESSO BRASILEIRO DE FRUTICULTURA, 16. 2000, Fortaleza-CE. Anais..., Fortaleza: Embrapa Agroindústria Tropical/SBF, 2000. p.79.

TEIXEIRA, L.A.J.; SPIRONELLO, A..; QUAGGIO, J.A.; FURLANI, P.R. Banana. In: RAIJ, B. van; CANTARELLA, H.; QUAGGIO, J.A.; FURLANI, A.M.C., ed. Recomendações de adubação e calagem para o Estado de São Paulo. 2.ed. rev. e atual. Campinas: Instituto Agronômico/Fundação IAC, 1997. p.131-132. (Boletim Técnico, $100)$. 